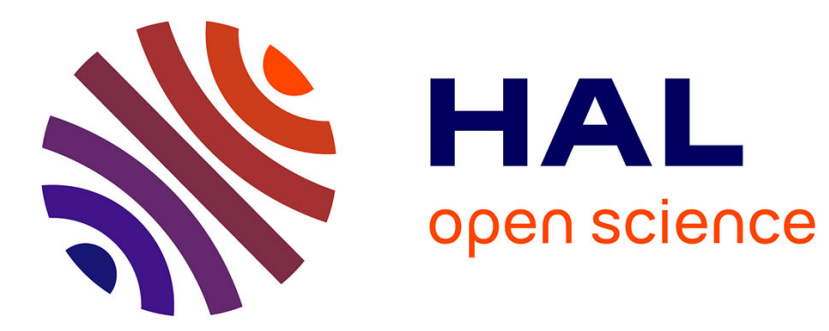

\title{
Acoustic wave propagation in effective graded fully anisotropic fluid layers
}

Théo Cavalieri, Jean Boulvert, Logan Schwan, Gwenael Gabard, Vicent

Romero-Garcia, Jean-Philippe Groby, Marie Escouflaire, Jacky Mardjono

\section{- To cite this version:}

Théo Cavalieri, Jean Boulvert, Logan Schwan, Gwenael Gabard, Vicent Romero-Garcia, et al.. Acoustic wave propagation in effective graded fully anisotropic fluid layers. Journal of the Acoustical Society of America, 2019, 146 (5), pp.3400-3408. 10.1121/1.5131653 . hal-02385196

\section{HAL Id: hal-02385196 https://hal.science/hal-02385196}

Submitted on 28 Nov 2019

HAL is a multi-disciplinary open access archive for the deposit and dissemination of scientific research documents, whether they are published or not. The documents may come from teaching and research institutions in France or abroad, or from public or private research centers.
L'archive ouverte pluridisciplinaire HAL, est destinée au dépôt et à la diffusion de documents scientifiques de niveau recherche, publiés ou non, émanant des établissements d'enseignement et de recherche français ou étrangers, des laboratoires publics ou privés. 


\section{Acoustic wave propagation in effective graded fully-anisotropic fluid layers}

Théo Cavalieri, ${ }^{1,2, a)}$ Jean Boulvert, ${ }^{1,2,3}$ Logan Schwan, ${ }^{1}$ Gwénaël Gabard, ${ }^{1}$ Vicent

Romero-Garcia, ${ }^{1}$ Jean-Philippe Groby, ${ }^{1}$ Marie Escouflaire, ${ }^{2}$ and Jacky Mardjono ${ }^{2}$

${ }^{1}$ Laboratoire d'Acoustique de l'Université du Mans, LAUM - UMR CNRS 6613,

Le Mans Université, Avenue Olivier Messiaen, 72085 LE MANS CEDEX 9,

France

${ }^{2}$ Safran Aircraft Engines, Rond Point René Ravaud-Réau, 77550 Moissy-Cramayel,

France

${ }^{3}$ Laboratoire d'Analyse Vibratoire et Acoustique, LAVA,

Department of Mechanical Engineering, École Polytechnique de Montréal,

P.O. Box 6079 Station Centre-ville, Montréal, Québec H3C 3A\%,

Canada

(Dated: 3 September 2019) 
This work deals with the modeling of sound wave propagation in anisotropic and heterogeneous media. The scattering problem considered in this work involves an infinite layer of finite thickness containing an anisotropic fluid whose properties can vary along the depth of the layer. The specular transmission and reflection of an acoustic plane wave by such a layer is modeled through the state vector formalism for the acoustic fields. This is solved using three different numerical techniques, namely the transfer matrix method, Peano series and the transfer Green's function. These three methods are compared to demonstrate the convergence of the numerical solutions. Moreover, the implemented numerical procedures allow to retrieve the internal acoustic fields and show their dependency along with the fluid's anisotropic properties. Results are then presented to illustrate the changes in absorption that can be achieved by tuning the anisotropy of the fluid as well as the variation of these properties across the depth of the layer. The results presented are in very good agreement across the different methods. Given that many porous materials can be modeled as equivalent fluids, the results presented show the potential offered by such numerical techniques, and can further give more insight on inhomogeneous anisotropic porous materials.

Keywords: acoustic control, anisotropic fluid, heterogeneous fluid, graded porous layer, absorption

\footnotetext{
a) theo.cavalieri@univ-lemans.fr
} 


\section{INTRODUCTION}

Acoustic treatments involving porous materials are commonly used for sound absorption purposes. The recent development of additive manufacturing provides more control on the micro-structures of these porous materials. Hence, the anisotropic and graded properties of such microstructures influence the wave propagation in the medium, which is numerically described. A rigid-frame porous medium is usually modeled as an equivalent fluid, that can display anisotropic and heterogeneous frequency dependent effective properties. One way to describe these effective properties is the well-known Johnson-Champoux-Allard-Lafarge (JCAL) model $^{1}$ which provides the thermal and viscous dynamic permeabilities of the propagation medium. For a periodic porous material, formed by a repetition of a unit cell, the JCAL model can rely on homogenized properties of this unit cell calculated using the method of multiple scales ${ }^{2}$. Since the viscous dissipation has been shown to be directiondependent $^{3-5}$ in anisotropic media, the same considerations are used in the current paper. Recent work ${ }^{6}$ have shown that anisotropic materials can have different apparent sound speed depending on the direction of propagation, coupling viscous and inertial regimes. This is especially visible at grazing angles of incidence, and can be exploited for absorption considering a diffuse field where all incidences are accounted for. The derivation of the equations has been done recently to retrieve the effective properties of an anisotropic homogeneous material $^{7}$, and is recalled in the section regarding wave propagation.

The present work focuses on the modeling and analysis of inhomogeneous anisotropic materials. The scattering problem considered here involves an infinite layer of finite thickness 
containing an anisotropic fluid whose properties can vary across the depth of the layer. The transmission and reflection of an acoustic plane wave by such a layer is modeled through the state vector formalism, which is solved using three different techniques. First, the layer is assumed piece-wise constant and the standard transfer matrix method (TMM) ${ }^{8}$ is employed. The other two methods are applicable to continuously graded media. The Peano Series (PS) has previously been used for graded $^{9-11}$ and anisotropic materials ${ }^{12}$, and wave-splitting techniques for continuously graded media ${ }^{13-18}$. In addition, the internal fields and dissipation rate of energy are estimated ${ }^{19}$ and shown to be dependent on the fluid's effective properties. Other solution procedures can however be applied to approximate such propagation problem, as Euler or Runge-Kutta iterative schemes, which are commonly used for linear systems ${ }^{14}$.

The article is organized as follows, we first introduce the equivalent fluid model and the propagation problem considered in this work. The different numerical approaches are then presented, so as to solve for the acoustic fields inside the layer. Numerical results of the scattering coefficients on such anisotropic graded material are presented for all the methods considered, which show good agreement. Finally, further insight is provided into the dissipation rate within the anisotropic material and in the role played by the orientation of the micro-structure.

\section{PROPAGATION IN GRADED ANISOTROPIC FLUID LAYERS}

In this section the propagation of a plane wave through an anisotropic, heterogeneous equivalent fluid is described. We set the reference in the Cartesian coordinate system $\mathcal{R}_{0}=$ 
$\left(O, \mathbf{e}_{1}, \mathbf{e}_{2}, \mathbf{e}_{3}\right)$ with the associated spatial coordinates vector $\mathbf{x}=\left(x_{1}, x_{2}, x_{3}\right) \in \mathbb{R}^{3}$. The fluid layer, denoted $\Omega$, is a slab of finite thickness $L$ and infinite extent in the $\left(0, \mathbf{x}_{\perp}\right)$ plane, as illustrated in Fig. 1. The subscript $\perp$ denotes the restriction of a vector to the $\left(O, \mathbf{x}_{\perp}\right)$ plane with $\mathbf{x}_{\perp}=\left\{x_{1}, x_{2}\right\}$. The domain $\Omega$ is delimited by the plane boundaries at $x_{3}=0$ and $x_{3}=L$ denoted $\Gamma_{0}$ and $\Gamma_{L}$ respectively. We solve for the sound field in this layer $\Omega$ in the linear harmonic regime using the time convention $\mathrm{e}^{-\mathrm{i} \omega t}$ where $\omega$ is the angular frequency. The effective bulk modulus and density of the anisotropic heterogeneous fluid are denoted $B\left(x_{3}, \omega\right)$ and $\boldsymbol{\rho}\left(x_{3}, \omega\right)$. Note that these quantities are complex-valued, frequency dependent and can vary along the $x_{3}$ direction, moreover, while the bulk modulus of the medium is scalar, the density is a second order tensor accounting for anisotropic phenomena. The pressure $p$ and velocity $\mathbf{v}$ induced by the acoustic field in $\Omega$ are governed by the following linear equations for mass conservation and momentum conservation

$$
\begin{aligned}
\mathrm{i} \omega \boldsymbol{\rho}\left(x_{3}, \omega\right) \mathbf{v}(\mathbf{x}, \omega) & =\nabla p(\mathbf{x}, \omega) \\
\mathrm{i} \omega B^{-1}\left(x_{3}, \omega\right) p(\mathbf{x}, \omega) & =\nabla \cdot \mathbf{v}(\mathbf{x}, \omega) .
\end{aligned}
$$

The exterior of the domain $\Omega$ is denoted $\Omega_{0}$ and contains an homogeneous isotropic fluid, taken to be air in this case. The density of air is $\rho_{0}=1.213 \mathrm{~kg} \cdot \mathrm{m}^{-3}$ and its bulk modulus $B_{0}=\gamma P_{0}$ with $\gamma=1.4$ the ratio of specific heat and $P_{0}=101325 \mathrm{~Pa}$ the atmospheric pressure. The sound field in the exterior domain $\Omega_{0}$ satisfies

$$
\begin{aligned}
\mathrm{i} \omega \rho_{0} \mathbf{v}(\mathbf{x}, \omega) & =\nabla p(\mathbf{x}, \omega) \\
\mathrm{i} \omega B_{0}^{-1} p(\mathbf{x}, \omega) & =\nabla \cdot \mathbf{v}(\mathbf{x}, \omega) .
\end{aligned}
$$


While the density of the isotropic fluid in $\Omega_{0}$ is described by the scalar $\rho_{0}$, the anisotropy

of the fluid in the layer $\Omega$ is described by the tensor density $\boldsymbol{\rho}$. This tensor accounts for the fact that the properties of the waves in $\Omega$ depend on the direction of propagation. The density tensor $\rho$ is diagonal in the special case where its principal directions are aligned with the coordinate system $\mathcal{R}_{0}$. But in the general case it is full, symmetric and can be written

$$
\boldsymbol{\rho}=\mathbf{R}\left[\begin{array}{ccc}
\rho_{11} & 0 & 0 \\
0 & \rho_{22} & 0 \\
0 & 0 & \rho_{33}
\end{array}\right]_{\mathcal{R}_{\Omega}} \mathbf{R}^{T}
$$

with $\mathbf{R}$ the complete rotation matrix accounting for the yaw, pitch and roll angles, respectively $\left(u_{1}, u_{2}, u_{3}\right)$ along $\left(\mathbf{e}_{1}, \mathbf{e}_{2}, \mathbf{e}_{3}\right)$. For the sake of simplicity and since the particle velocity depends on the inverse of the density tensor, the second-order tensor $\mathbf{H}=\boldsymbol{\rho}^{-1}$ remains symmetric and will be used instead of $\boldsymbol{\rho}$ in the remainder of this work.

In the upper region of $\Omega_{0}, x_{3} \geq L$, we define an incident plane wave with unit amplitude:

$$
p^{i}(\mathbf{x}, \omega)=\mathrm{e}^{\mathrm{i} k_{1} x_{1}+\mathrm{i} k_{2} x_{2}-\mathrm{i} k_{3}\left(x_{3}-L\right)},
$$

where the components of the wave-vector $\mathbf{k}^{i}$ are given by

$$
\begin{aligned}
& k_{1}=-k_{0} \cos (\theta) \cos (\psi), \\
& k_{2}=-k_{0} \cos (\theta) \sin (\psi), \\
& k_{3}=k_{0} \sin (\theta),
\end{aligned}
$$

with $\psi$ and $\theta$ the polar and elevation angles, respectively. $k_{0}=\omega / c_{0}$ is the free-field acoustic wave-number. 
The presence of the anisotropic layer $\Omega$ gives rise to a reflected wave $p^{r}$ in the upper region of $\Omega_{0}$ and to a transmitted wave $p^{t}$ in the lower region of $\Omega_{0}, x_{3} \leq 0$. These are written

$$
\begin{aligned}
& p^{r}(\mathbf{x}, \omega)=\tilde{R} \mathrm{e}^{\mathrm{i} \mathbf{k}_{\perp} \cdot \mathbf{x}_{\perp}+\mathrm{i} k_{3}\left(x_{3}-L\right)}, \\
& p^{t}(\mathbf{x}, \omega)=\tilde{T} \mathrm{e}^{\mathrm{i} \mathbf{k}_{\perp} \cdot \mathbf{x}_{\perp}-\mathrm{i} k_{3} x_{3}},
\end{aligned}
$$

where $\tilde{R}$ and $\tilde{T}$ are the specular coefficients of reflection and transmission and $\mathbf{k}_{\perp}=\left\{k_{1}, k_{2}\right\}$ and $\mathbf{x}_{\perp}=\left\{x_{1}, x_{2}\right\}$. As the incident wave could physically come from $x_{3}<0$, it is important to be explicit about the scattering coefficients which are $\tilde{R}^{ \pm}$and $\tilde{T}^{ \pm}$, depending on the sign of wave incidence. The system being reciprocal we reach $\tilde{T}=\tilde{T}^{+}=\tilde{T}^{-}$, whereas the distinction has to be made for the reflection since the heterogeneity of the medium can be non-symmetric. Without any specific considerations about the effective properties of the medium, $\tilde{R}^{+} \neq \tilde{R}^{-}$in the inhomogeneous case. For the sake of simplicity and as reversing the layer $\Omega$ between its interfaces is equivalent to propagating in the opposite direction, we use the notation $\tilde{R}=\tilde{R}^{+}$when the incident waves comes from the upper region $x_{3} \geq L$. However, the solution procedures developed further are valuable for all scattering coefficients.

The incident plane wave $p^{i}$ also induces a sound field in the anisotropic and graded layer $\Omega$. Given that (i) the properties of this layer are independent of $x_{1}$ and $x_{2}$ and (ii) the incident field has an harmonic spatial dependence $e^{\mathbf{i} \mathbf{k}_{\perp} \cdot \mathbf{x}_{\perp}}$, it is clear that the wave field in 


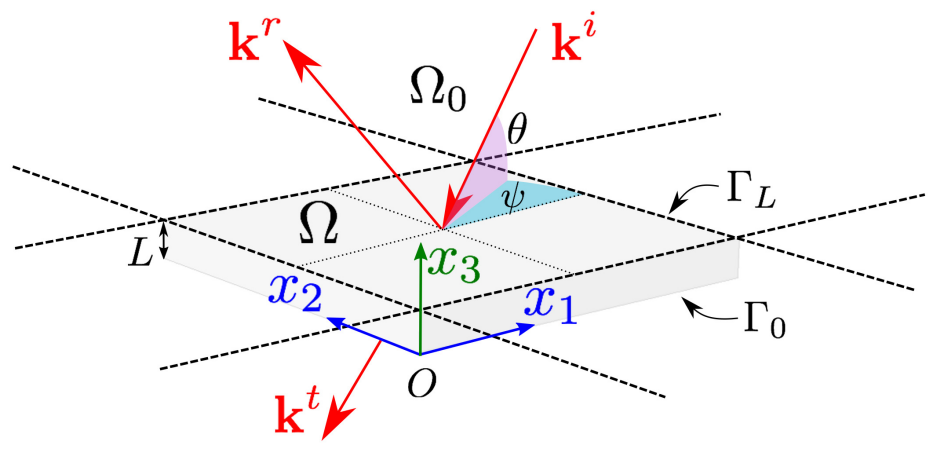

FIG. 1. [Color online] Schematic representation of the propagation problem in $\Omega_{0}$ and $\Omega$. A fluid layer of finite thickness $L$ along $x_{3}$, with infinite dimension in the $\left(O, \mathbf{x}_{\perp}\right)$ plane and interfaces $\Gamma_{0}$ and $\Gamma_{L}$. Incident, reflected and transmitted wave-vectors are represented with red arrows. The elevation and azimuthal angles $\theta$ and $\psi$ are shown respectively in purple and cyan.

the layer $\Omega$ retains the same harmonic spatial dependence:

$$
\begin{gathered}
p(\mathbf{x}, \omega)=p\left(x_{3}\right) \mathrm{e}^{\mathrm{i} \mathbf{k}_{\perp} \cdot \mathbf{x}_{\perp}} \\
\mathbf{v}(\mathbf{x}, \omega)=\mathbf{v}\left(x_{3}\right) \mathrm{e}^{\mathrm{i} \mathbf{k}_{\perp} \cdot \mathbf{x}_{\perp}}
\end{gathered}
$$

The derivation of the governing equations Eqs. (1) has recently been done for retrieval techniques and applied to fully-anisotropic porous materials ${ }^{7}$. The process is recalled as follows, and leads to the state-vector equation for pressure and normal particle velocity. From the conservation equations Eqs. (1), the transverse and normal components of the 
fields are expanded,

$$
\begin{aligned}
\mathrm{i} \omega \mathbf{v}_{\perp} & =\mathrm{i} \mathbf{H}_{\perp} \cdot \mathbf{k}_{\perp} p+H_{33} \mathbf{q} \frac{\partial p}{\partial x_{3}}, \\
\mathrm{i} \omega H_{33}^{-1} v_{3} & =\mathrm{i} \mathbf{k}_{\perp} \cdot \mathbf{q} p+\frac{\partial p}{\partial x_{3}}, \\
\mathrm{i} \omega B^{-1} p & =\mathrm{i} \mathbf{k}_{\perp} \cdot \mathbf{v}_{\perp}+\frac{\partial v_{3}}{\partial x_{3}}
\end{aligned}
$$

84

where we have again used the notation $\mathbf{v}_{\perp}=\left\{v_{1}, v_{2}\right\}$. We have also introduced the coupling vector $\mathbf{q}=\left\{H_{13} / H_{33}, H_{23} / H_{33}\right\}$ and the $2 \times 2$ matrix $\mathbf{H}_{\perp}=H_{m n} \forall(m, n) \in\{1,2\}^{2}$. From Eqs. (10a) and (10c) we get

$$
\mathrm{i} \omega B^{-1} p=\mathrm{i} \mathbf{k}_{\perp} \cdot\left[\mathrm{i}\left(\mathbf{H}_{\perp} \cdot \mathbf{k}_{\perp}\right) \frac{p}{\mathrm{i} \omega}+\frac{H_{33}}{\mathrm{i} \omega} \mathbf{q} \frac{\partial p}{\partial x_{3}}\right]+\frac{\partial v_{3}}{\partial x_{3}} .
$$

Together with the momentum conservation in Eq. (10b), leads to

$$
\mathrm{i} \omega B^{-1} p=H_{33}\left(\mathbf{k}_{\perp} \cdot \mathbf{q}\right)^{2} \frac{p}{\mathrm{i} \omega}-\mathbf{k}_{\perp} \cdot\left(\mathbf{H}_{\perp} \cdot \mathbf{k}_{\perp}\right) \frac{p}{\mathrm{i} \omega}+\mathrm{i}\left(\mathbf{k}_{\perp} \cdot \mathbf{q}\right) v_{3}+\frac{\partial v_{3}}{\partial x_{3}}
$$

where after rearranging the pressure terms, emerges the equivalent bulk modulus:

$$
B_{e q}^{-1}=B^{-1}+\left[H_{33}\left(\mathbf{k}_{\perp} \cdot \mathbf{q}\right)^{2}-\mathbf{k}_{\perp} \cdot\left(\mathbf{H}_{\perp} \cdot \mathbf{k}_{\perp}\right)\right] / \omega^{2}
$$

Yields the following equation of mass conservation, where $B_{e q}$ relates the compressibility effects of the equivalent fluid, accounting for anisotropic dependencies and oblique considerations,

$$
\mathrm{i} \omega B_{e q}^{-1} p=\mathrm{i} \mathbf{k}_{\perp} \cdot \mathbf{q} v_{3}+\frac{\partial v_{3}}{\partial x_{3}}
$$

and with Eq. 10b, they characterize the sound field in the layer $\Omega$, with equivalent density $H_{33}^{-1}$ and bulk modulus $B_{e q}$. They can be written using a state-vector formulation

$$
\frac{\mathrm{d} \mathbf{W}}{\mathrm{d} x_{3}}=\mathbf{A}\left(x_{3}\right) \mathbf{W}
$$


where we have introduced the state vector $\mathbf{W}=\left\{p, v_{3}\right\}^{T}$ (with ${ }^{T}$ the non-conjugate transpose), and the matrix

$$
\mathbf{A}\left(x_{3}\right)=\left[\begin{array}{cc}
-\mathrm{i} \mathbf{k}_{\perp} \cdot \mathbf{q} & \mathrm{i} \omega H_{33}^{-1} \\
\mathrm{i} \omega B_{e q}^{-1} & -\mathrm{i} \mathbf{k}_{\perp} \cdot \mathbf{q}
\end{array}\right]
$$

At the interfaces $\Gamma_{0}$ and $\Gamma_{L}$ between the anisotropic layer and the surrounding fluid, the continuity of pressure and normal velocity is imposed as boundary conditions. As a consequence, the state vector at both interfaces reads

$$
\mathbf{W}_{L}=\left\{\begin{array}{c}
1+\tilde{R} \\
Z_{e}^{-1}(\tilde{R}-1)
\end{array}\right\} \text { and } \quad \mathbf{W}_{0}=\left\{\begin{array}{c}
\tilde{T} \\
-Z_{e}^{-1} \tilde{T}
\end{array}\right\}
$$

with $Z_{e}=Z_{0} / \sin (\theta)$ the apparent impedance of the air in domain $\Omega_{0}$ with respect to the unit outward normal vector $\mathbf{n}=\mathbf{e}_{3}$ at interface $\Gamma_{L}$. Note that in the case where the layer is rigidly backed (absorption problem), the boundary term at $\Gamma_{0}$ simplifies to $\mathbf{W}_{0}=\{p(0), 0\}^{T}$ since the Neumann condition involves zero normal velocity on the rigid layer backing.

\section{SOLUTION PROCEDURES}

The state-vector Eq. (15) can be solved using a variety of numerical techniques. In this section three different methods are presented. The well-known TMM is first described, then two other approaches are presented for continuously graded media. 
with $l_{i}=x_{3}^{(i+1)}-x_{3}^{(i)}$. This expression can be directly written as a matrix exponential ${ }^{20}$ :

$$
\mathbf{M}\left(x_{3}^{(i+1)}, x_{3}^{(i)}\right)=\exp \left(\mathbf{A}_{i} l_{i}\right)
$$

where $\mathbf{M}$ is the matricant which can be written in terms of the constant matrix $\mathbf{A}_{i}$ associated with the $i$ th homogeneous layer:

$$
\mathbf{A}_{i}=\mathbf{A}\left(\frac{x_{3}^{(i+1)}+x_{3}^{(i)}}{2}\right)
$$

To do so we first diagonalize this matrix by writing $\mathbf{A}_{i}=\mathbf{V}_{i}^{-1} \boldsymbol{\lambda}_{i} \mathbf{V}_{i}$ with $\boldsymbol{\lambda}_{i}$ the diagonal matrix of eigenvalues and $\mathbf{V}_{i}$ the matrix of eigenvectors. The state-vector formulation Eq. (15) in the $i$ th layer can be transformed into two decoupled ordinary differential equations:

$$
\frac{\mathrm{d}}{\mathrm{d} x_{3}}\left(\mathbf{V}_{i} \mathbf{W}\right)=\boldsymbol{\lambda}_{i}\left(\mathbf{V}_{i} \mathbf{W}\right)
$$

These can be readily solved to obtain the matricant:

$$
\mathbf{M}\left(x_{3}^{(i+1)}, x_{3}^{(i)}\right)=\mathbf{V}_{i}^{-1}\left(\begin{array}{cc}
\mathrm{e}^{\lambda_{1} l_{i}} & 0 \\
0 & \mathrm{e}^{\lambda_{2} l_{i}}
\end{array}\right) \mathbf{V}_{i}
$$


The overall transfer matrix $\mathbf{M}$ relating the state vectors at the two interfaces $\Gamma_{0}$ and $\Gamma_{L}$ is defined as the product of the matricants of all the homogeneous layers:

$$
\mathbf{W}_{L}=\mathbf{M W}_{0}, \quad \mathbf{M}=\prod_{i=0}^{N-1} \mathrm{e}^{\mathbf{A}_{i} l_{i}}
$$

The discretization of domain $\Omega$ is chosen to be linear across $N=40$ positions, and will serve as comparison with two different methods which follow.

\section{B. Peano Series}

Another approach to solve Eq. (15) is to use the PS which have previously been used for continuously graded isotropic materials ${ }^{9}$. In the homogeneous case, i.e. when $\mathbf{A}$ is constant, the PS can be shown to be equivalent to the product of matrix exponentials in Eq. (23). In the present case of $x_{3}$ dependent properties, the matrix $\mathbf{A}$ does not commute with itself for different values of $x_{3}$, so $\forall\left(x_{3}^{\prime}, x_{3}^{\prime \prime}\right) \in[0, L]^{2}, x_{3}^{\prime} \neq x_{3}^{\prime \prime},\left[\mathbf{A}\left(x_{3}^{\prime}\right) \mathbf{A}\left(x_{3}^{\prime \prime}\right)-\mathbf{A}\left(x_{3}^{\prime \prime}\right) \mathbf{A}\left(x_{3}^{\prime}\right)\right] \neq 0$ and the matricant is no longer defined by matrix exponentials, but rather by the Peano series. Using this formalism, the matricant $\mathbf{M}$ defined by Eq. (23) is written as an infinite series of integrals ${ }^{18,20}$ :

$$
\mathbf{M}\left(0, x_{3}\right)=\mathbb{I}_{\mathrm{d}}+\int_{0}^{x_{3}} \mathbf{A}(\xi) \mathrm{d} \xi+\int_{0}^{x_{3}} \mathbf{A}(\xi) \int_{0}^{\xi} \mathbf{A}\left(\xi_{1}\right) \mathrm{d} \xi \mathrm{d} \xi_{1}+\ldots
$$

In practice this is calculated through the use of the following recurrence relations ${ }^{11}$,

$$
\left\{\begin{array}{l}
\mathbf{M}^{\{0\}}(0, L)=\mathbb{I}_{\mathrm{d}} \\
\mathbf{M}^{\{n\}}(0, L)=\mathbb{I}_{\mathrm{d}}+\int_{0}^{L} \mathbf{A}\left(x_{3}\right) \mathbf{M}^{\{n-1\}}\left(x_{3}\right) \mathrm{d} x_{3}
\end{array}\right.
$$


and the state vector relation at both interfaces now reads,

$$
\mathbf{W}_{L}=\lim _{n \rightarrow \infty} \mathbf{M}^{\{n\}}(0, L) \mathbf{W}_{0}
$$

An approximate solution is obtained by truncating this infinite series. In fact, unlike the TMM where the matricant of the system is assembled piece by piece, each term of the integral series accounts for the whole domain $0<x_{3}<L$. The integral itself is estimated by the trapezoidal method at each iteration, using the same unit spacing $L / N$. Hence, any additional term of the truncated series tends to refine the solution given by this method. The recurrence relation is chosen to be expanded up to 50 terms, a sufficient number for the series to converge.

\section{Wave-Splitting, Transfer Green Functions}

The wave-splitting method relies on the separation of the overall acoustic field into forward and backward propagative waves ${ }^{14,18}$. Since the effective properties of the medium are inhomogeneous along $x_{3}$, the wave-splitting applied in the current paper is not related to $\Omega$, but rather with respect to the domain $\Omega_{0}{ }^{15,16}$. The wave-splitting matrix is independent of the graded parameters (tensorial density $\boldsymbol{\rho}$ and equivalent bulk modulus $B_{e q}$ ), which ensures the split fields to be continuous across any $x_{3}$-plane in the medium $\Omega^{17}$. These are defined as $s^{ \pm}=\left(p \pm Z_{e} \mathbf{v} \cdot \mathbf{n}\right) / 2$ where the \pm sign indicates the direction of propagation relative to the unit vector $\mathbf{n}$. Although they only have a physical sense in $\Omega_{0}$ according to the wavesplitting transformation, the associated change of basis remains valid. It is then possible to 
introduce a new vector $\mathbf{S}=\left\{s^{+}, s^{-}\right\}^{T}$ which is related to the original vector $\mathbf{W}$ by,

$$
\mathbf{S}\left(x_{3}, \omega\right)=\mathbf{Z W}\left(x_{3}, \omega\right), \quad \text { with } \mathbf{Z}=\frac{1}{2}\left[\begin{array}{cc}
1 & Z_{e} \\
1 & -Z_{e}
\end{array}\right]
$$

Introducing this definition in the state vector formulation Eq. (15), it is straightforward to obtain:

$$
\frac{\mathrm{d}}{\mathrm{d} x_{3}} \mathbf{S}=\mathbf{B}\left(x_{3}\right) \mathbf{S}
$$

with

$$
\mathbf{B}\left(x_{3}\right)=\mathbf{Z A}\left(x_{3}\right) \mathbf{Z}^{-1}=\left[\begin{array}{cc}
U^{+} & U^{-} \\
-U^{-} & -U^{+}
\end{array}\right]-\mathrm{i}\left(\mathbf{k}_{\perp} \cdot \mathbf{q}\right) \mathbb{I}_{\mathrm{d}},
$$

$\mathbb{I}_{\mathrm{d}}$ being the identity matrix, and

$$
U^{ \pm}\left(x_{3}, \omega\right)=\frac{i \omega}{2}\left[Z_{e} B_{e q}^{-1}\left(x_{3}, \omega\right) \pm H_{33}^{-1}\left(x_{3}, \omega\right) Z_{e}^{-1}\right]
$$

The differential equations Eq. (28) can be solved using the transfer Green's functions $(\mathrm{TGF})^{19}$ method by writing the forward and backward internal fields in terms of the transmitted wave $s^{-}(0, \omega)$ as follows:

$$
s^{ \pm}\left(x_{3}, \omega\right)=G^{ \pm}\left(x_{3}, \omega\right) s^{-}(0, \omega)
$$

where $G^{ \pm}$denote the two Green's functions. They are solutions of the following differential equations

$$
\frac{\mathrm{d} \mathbf{G}}{\mathrm{d} x_{3}}=\mathbf{B G},
$$

with $\mathbf{G}=\left\{G^{+}, G^{-}\right\}$ 
In the case of an absorption problem (rigid backing at $\Gamma_{0}$ ) the boundary condition for the Green functions Eq. (30) reads $\mathbf{G}_{0}=\{1,1\}$ as a total specular reflection. In the case of a transmission problem, we must have a total transmission at the interface $\Gamma_{0}$, corresponding to $\mathbf{G}_{0}=\{1,0\}$. The fluid layer heterogeneity being of macroscopic scale (the order of $\mathrm{L}$ ), the spatial discretization is easily achieved. The continuous graded properties along $x_{3}$ in the domain $\Omega$ are split linearly into $N=40$ positions. The differential system of equations, Eq. (30) is solved numerically.

\section{RESULTS AND DISCUSSIONS}

This section deals with the numerical validation of the proposed models. The scattering coefficients are retrieved with all three different methods and applied to an heterogeneous anisotropic porous material.

\section{A. Scattering coefficients}

With the TMM and the PS the reflection and transmission coefficients are readily available as part of the solution procedures. From the relation $\mathbf{W}_{L}=\mathbf{M} \mathbf{W}_{0}$ from Eq. (23) one can derive the following expressions for these coefficients:

$$
\begin{aligned}
& \tilde{T}=2 Z_{e}^{-1}\left[Z_{e}^{-1} \operatorname{Tr}(\mathbf{M})-Z_{e}^{-2} M_{12}-M_{21}\right]^{-1}, \\
& \tilde{R}=M_{11} \tilde{T}-Z_{e}^{-1} M_{12} \tilde{T}-1
\end{aligned}
$$


where $\operatorname{Tr}(\mathbf{M})$ is the trace of the square matrix $\mathbf{M}$ and $M_{i j}$ are the coefficients of the matrix. Note that $\tilde{R}$ and $\tilde{T}$ are functions of the angular frequency $\omega$ and the incidence angles (the polar and elevation angles $\psi$ and $\theta$, respectively).

For the wave-splitting method, the reflection and transmission coefficients are recovered from the solutions for the Green's functions $G^{+}$and $G^{-}$as follows ${ }^{14,17,18}$ :

$$
\begin{aligned}
& \tilde{T}=1 / G^{-}(0), \\
& \tilde{R}=G^{+}(L) / G^{-}(L) .
\end{aligned}
$$

To quantify the acoustic dissipation inside the layer $\Omega$ we calculate the absorption coefficient. As mentioned earlier, as the scattering coefficients depend from the direction of incidence, the absorption coefficient follows the same dependency,

$$
\alpha^{ \pm}(\omega)=1-\left|\tilde{R}^{ \pm}(\omega)\right|^{2}-|\tilde{T}(\omega)|^{2} .
$$

It will vary between 0 and 1 and can also be calculated when the layer is rigidly backed so $\tilde{T}=0$. The different computing methods have been compared to the transfer matrix method. For a similar spatial sampling (linear with $N=40$ ) the relative error between each method is below $0.2 \%$ and the average computation time per frequency is $t_{t g f} \approx 0.21 \mathrm{~s}$ for Green's functions (and mainly depends on absolute and relative tolerances of the numerical integration), while $t_{p s} \approx 0.05 \mathrm{~s}$ for 50 terms of Peano Series and $t_{t m m} \leq 0.01 \mathrm{~s}$ for TMM. These results are obtained by averaging the computing time over 100 frequency points, the overall comparison for the three different methods can also be done in parallel. Moreover, other numerical differentiation procedures can be set up to reach the scattering coefficients, such as Runge-Kutta schemes ${ }^{14}$. 
TABLE I. Homogenized JCAL parameters for the anisotropic unit cell with characteristic size $\ell_{c}$ in the coordinate system $\mathcal{R}_{0}$.

\begin{tabular}{|c|c|c|c|c|c|c|}
\hline & $\phi(1)$ & $\Lambda^{\prime}(\mathrm{m})$ & $\mathcal{K}_{0}^{\prime}\left(\mathrm{m}^{2}\right)$ & $\tau^{\infty}(1)$ & $\Lambda(\mathrm{m})$ & $\mathcal{K}_{0}\left(\mathrm{~m}^{2}\right)$ \\
\hline$\Omega$ & 0.7210 & $0.533 \ell_{c}$ & $0.0214 \ell_{c}^{2}$ & - & - & - \\
\hline $\mathbf{e}_{1}$ & - & - & - & 2.987 & $0.129 \ell_{c}$ & $5.7410^{-4} \ell_{c}^{2}$ \\
\hline $\mathbf{e}_{2}$ & - & - & - & 1.089 & $0.448 \ell_{c}$ & $1.5610^{-2} \ell_{c}^{2}$ \\
\hline $\mathbf{e}_{3}$ & - & - & - & 1.487 & $0.273 \ell_{c}$ & $4.8310^{-3} \ell_{c}^{2}$ \\
\hline
\end{tabular}

\section{B. Porous material}

The anisotropic fluid layer considered as an example in the present work is a periodic porous material. The unit cell that is periodically distributed to form this periodic material is a rigid cube of length $\ell_{c}$ from which an ellipsoid with semi-axes of different lengths is carved out, see Fig 2(c). The effective properties of this unit cell are obtained using the multiple-scale method outlined in Ref. 2 and 7. The resulting parameters of the JCAL model $^{1}$ are listed in Table I as functions of the unit cell size $\ell_{c}$ and in the coordinate system $\mathcal{R}_{0}$. Some of these parameters are scalar quantities (porosity $\phi$, characteristic thermal length $\Lambda^{\prime}$ and static thermal permeability $\mathcal{K}_{0}^{\prime}$ ) while others are tensorial (high-frequency tortuosity $\tau^{\infty}$, characteristic viscous length $\Lambda$ and static viscous permeability $\left.\mathcal{K}_{0}\right)$.

To obtain an inhomogeneous material the unit cell size $\ell_{c}$ is varied along the $x_{3}$ direction. As a consequence the effective JCAL parameters will also vary along this direction. The 
profile chosen as an example in this work is the 'ramp' shown in Fig. 2(a). The unit cell size $\ell_{c}$ is varied continuously from $0.1 \mathrm{~mm}$ at the base of the layer $\left(x_{3}=0\right)$ to $2 \mathrm{~mm}$ at the top of the layer $\left(x_{3}=L\right)$. This profile was chosen to achieve an impedance matching between the exterior domain and the porous material. The layer thickness is $L=50 \mathrm{~mm}$ and achieves perfect absorption at the frequency $f_{0}=2500 \mathrm{~Hz}$.

(a)
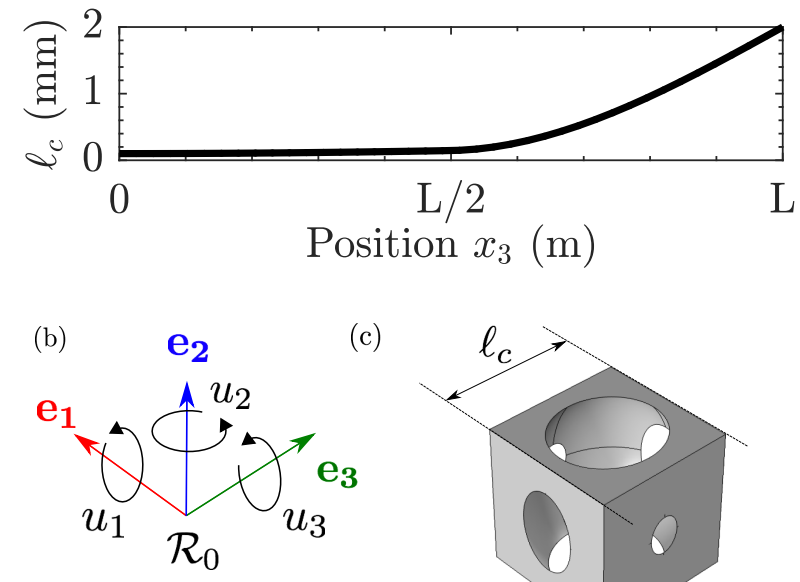

(c)

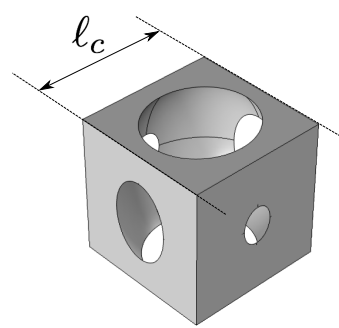

FIG. 2. [Color online] (a) Variation of the unit cell size $\ell_{c}$ along the depth of the porous material layer between $\Gamma_{0}$ and $\Gamma_{L}$. (b) Cartesian coordinate system $\mathcal{R}_{0}$ with its associated orthonormal basis $\left(\mathbf{e}_{1}, \mathbf{e}_{2}, \mathbf{e}_{3}\right)$ and the rotation angles $\left(u_{1}, u_{2}, u_{3}\right)$. (c) Unit cell for the periodic anisotropic porous material. Shown here is the rigid skeleton and a fluid region formed by a body-centered ellipsoid with semi-axes $r_{1}=0.51 \ell_{c}, r_{2}=0.7 \ell_{c}$ and $r_{3}=0.55 \ell_{c}$. 
(a)

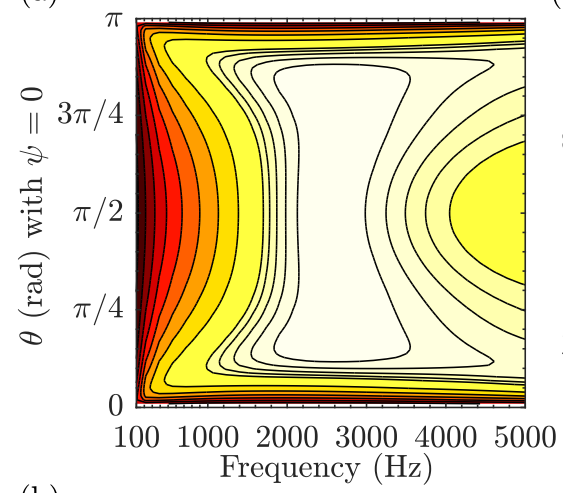

(b)

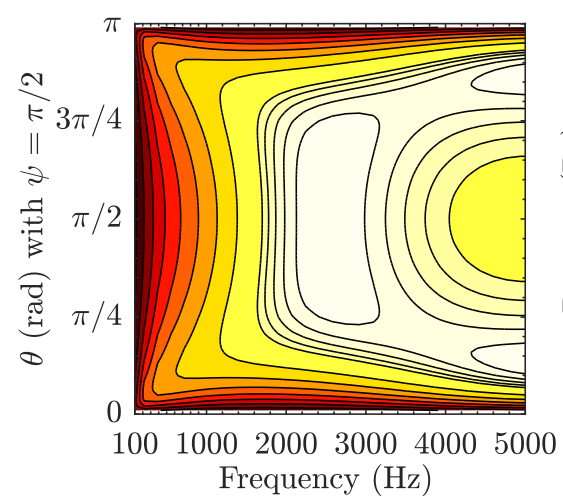

$\begin{array}{llllll}0 & 0.2 & 0.4 & 0.6 & 0.8 & 1\end{array}$ (c)

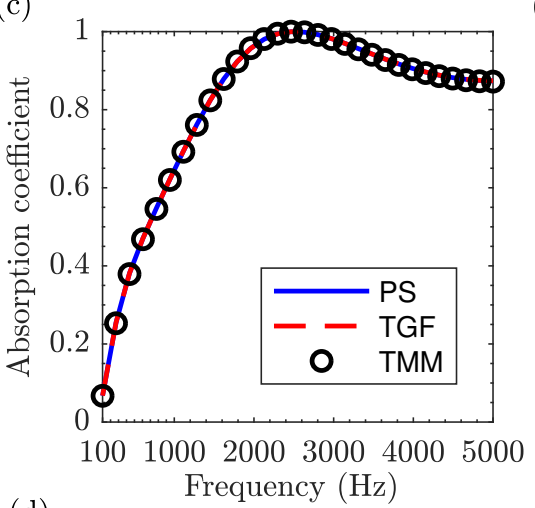

(d)

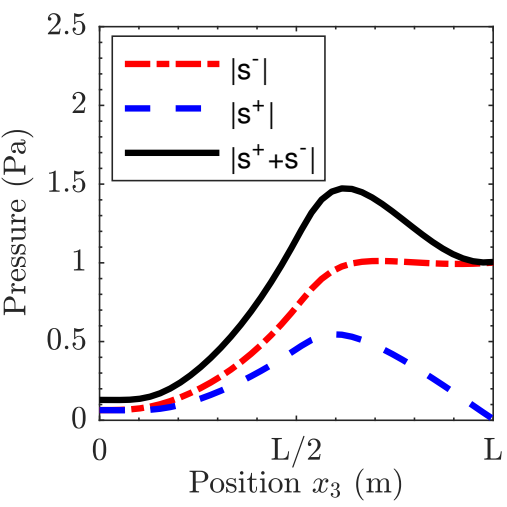

(e)

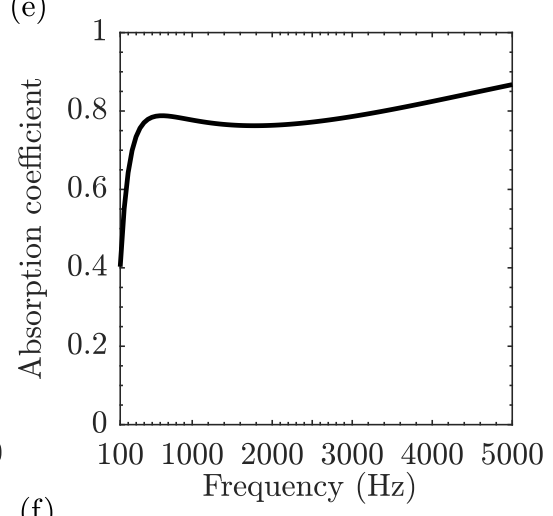

(f)

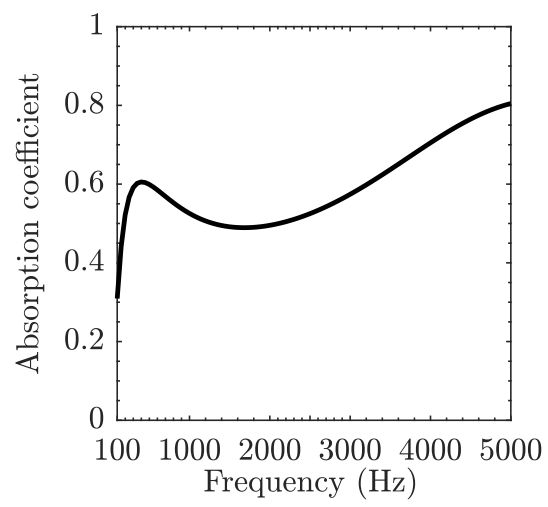

FIG. 3. [Color online] Absorption coefficient at oblique incidence, on the frequency range $100 \mathrm{~Hz}-5 \mathrm{kHz}$ and for elevation angle $\theta$ from 0 to $\pi$, with $\psi=0$ (a) and $\psi=\pi / 2$ (b). (c) Absorption coefficient at normal incidence, on the same frequency range, using the TMM, PS and TGF methods. (d) Magnitude of the split fields in the porous layer $\mathbf{S}$, for perfect absorption frequency $f_{0}$ at normal incidence. Absorption coefficient at grazing incidence, on the frequency range $100 \mathrm{~Hz}-5 \mathrm{kHz}$ and for elevation angle $\theta=\pi / 20$, with $\psi=0$ (e) and $\psi=\pi / 2$ (f).

\section{Influence of wave incidence}

We begin by considering the case of a plane wave at oblique incidence with $\mathbf{k}^{i}=$ $\left(k_{1}, k_{2}, k_{3}\right)$. Results are shown for an absorption problem, when the layer is rigidly backed 
at $\Gamma_{0}$. Fig. 3(a) shows the absorption coefficient as a function of frequency between $100 \mathrm{~Hz}$ and $5 \mathrm{kHz}$. The second axis spans the values of elevation angle, while the polar angle of incidence is $\psi=0$ in Fig.3(a) and $\psi=\pi / 2$ in Fig.3(b). While the absorption is limited at low frequency, this material is able to achieve a perfect absorption $(\alpha=1)$ for a frequency close to $f_{0}=2500 \mathrm{~Hz}$. However, we can observe a notable change in the absorption depending on the polar angle of incidence. Fig.3(c) also shows that the three solution procedures presented here (namely the TMM, PS and TGF) are in excellent agreement over the whole range of frequencies. Fig.3(d) shows the evolution of the forward and backward components $s^{ \pm}\left(x_{3}\right)$ in the layer $\Omega$ for the frequency where the perfect absorption $f_{0}$ is achieved. It is clear that the magnitude of the backward wave $s^{+}\left(x_{3}, \omega\right)=\left(p+Z_{e} v_{3}\right) / 2$ vanishes on the upper side of the layer $\left(x_{3}=L\right)$, which is consistent with the fact that there are no reflected wave at this frequency. Also visible in Fig.3(d) is the strong absorption of the forward propagating component $s^{-}$when it reaches the more resistive part of the porous layer (i.e. where $\ell_{c}$ is small). Concerning the dependence of $\alpha$ with the elevation angle $\theta$, the system tends towards total reflection for grazing incidences and the anisotropic properties of the fluid layer are clearly visible, as shown in Figs. 3(e) and 3(f).

\section{Effects of anisotropic coupling}

In the results above the unit cell has been aligned with the coordinate system, as shown in Fig.3. To illustrate the effects of the anisotropy of the material, one can rotate the unit cell using the expression given in Eq. (3). This is shown in Fig.4 for the absorption coefficient 
$\alpha$. Depending on the rotation components $\left(u_{1}, u_{2}, u_{3}\right)$ involved in the density tensor, the acoustic behavior of the fluid layer is significantly impacted, especially at high frequencies.

To provide further insight into the losses occurring within the layer, we derive the balance of acoustic energy in an anisotropic fluid. From the governing Eq. (1) in $\Omega$, one can derive,

$$
\begin{array}{r}
\mathrm{i} \omega \mathbf{v}^{*} \boldsymbol{\rho} \mathbf{v}-\mathbf{v}^{*} \cdot \nabla p=0, \\
\mathrm{i} \omega B^{-1}|p|^{2}-\bar{p} \nabla \cdot \mathbf{v}=0,
\end{array}
$$

where we have introduced the conjugated transposed velocity $\mathbf{v}^{*}$ and the conjugated pressure $\bar{p}$. As depicted in Eq.(3), the density tensor is complex and symmetric and emerges from the dynamic viscous permeability of the medium $\Omega$. It can be split into its complex components from the Toeplitz decomposition ${ }^{21}$ so, $\boldsymbol{\rho}=\boldsymbol{\rho}_{R}+\mathrm{i} \boldsymbol{\rho}_{I}$ with $\boldsymbol{\rho}_{R}=\left(\boldsymbol{\rho}+\boldsymbol{\rho}^{*}\right) / 2$ and $\boldsymbol{\rho}_{I}=$ $\left(\boldsymbol{\rho}-\boldsymbol{\rho}^{*}\right) / 2$ i. In the general case of a non-symmetric $\boldsymbol{\rho}$ tensor, both Hermitian matrices $\boldsymbol{\rho}_{R}$ and $\boldsymbol{\rho}_{I}$ remain complex-valued, however, in our case of symmetric tensor density, $\boldsymbol{\rho}_{R}$ and $\boldsymbol{\rho}_{I}$ are real. Taking the sum of both of the equations (33) yields,

$$
\frac{1}{2}(\mathrm{i} \omega)\left(\mathbf{v}^{*}\left(\boldsymbol{\rho}_{R}+\mathrm{i} \boldsymbol{\rho}_{I}\right) \mathbf{v}+B^{-1}|p|^{2}\right)=\frac{1}{2}\left(\mathbf{v}^{*} \cdot \nabla p+\bar{p} \nabla \cdot \mathbf{v}\right),
$$

which after expansion of the complex terms and reads,

$$
\frac{1}{2} \omega\left(\mathrm{i} \mathbf{v}^{*} \boldsymbol{\rho}_{R} \mathbf{v}-\mathbf{v}^{*} \boldsymbol{\rho}_{I} \mathbf{v}+\mathrm{i} B^{-1}|p|^{2}\right)=\frac{1}{2}\left(\mathbf{v}^{*} \cdot \nabla p+\bar{p} \nabla \cdot \mathbf{v}\right)
$$

Now considering the real part of this equality, it yields to the time average of the acoustic instantaneous intensity ${ }^{22}$, as the products $\mathbf{v}^{*} \boldsymbol{\rho}_{R} \mathbf{v}$ and $\mathbf{v}^{*} \boldsymbol{\rho}_{I} \mathbf{v}$ are real-valued,

$$
\frac{1}{2} \omega\left(\mathbf{v}^{*} \boldsymbol{\rho}_{I} \mathbf{v}+\operatorname{Im}\left\{B^{-1}\right\}|p|^{2}\right)=-\frac{1}{2} \operatorname{Re}\left\{\mathbf{v}^{*} \cdot \nabla p+\bar{p} \nabla \cdot \mathbf{v}\right\}
$$


where from the product rule of the divergence we now reach,

$$
\nabla \cdot\left(\frac{1}{2} \operatorname{Re}\{\mathcal{P}\}\right)=-\frac{1}{2} \omega\left(\mathbf{v}^{*} \boldsymbol{\rho}_{I} \mathbf{v}+\operatorname{Im}\left\{B^{-1}\right\}|p|^{2}\right)
$$

The left-hand side of this equation is the divergence of the Poynting vector $\mathcal{P}=p \mathbf{v}^{*}$, since the porous layer is purely lossy, we expect this term to be strictly negative. This quantity is homogeneous to the dissipation rate of acoustic energy at each infinitesimal point $x_{3} \in \Omega$ and is expressed in $\mathrm{W} \cdot \mathrm{m}^{-3}$. Although, it is estimated as only dependent of the normal direction $x_{3}$ since the acoustic fields in Eq. (9) show an harmonic spatial dependence.

It highlights the role of the coupling vector $\mathbf{q}$ and its effect on the fully-anisotropic behavior of such medium. The total energy lost in the system can be retrieved by spatial integration between boundaries $\Gamma_{0}$ and $\Gamma_{L}$. As all three components of the particle velocity are involved, the transverse part of $\mathbf{v}$ is derived from Eqs. (10a) and (10b).

Inside the domain $\Omega$, the transverse components of particle velocity read

$$
\mathbf{v}_{\perp}=\left(\mathbf{H}_{\perp} \cdot \mathbf{k}_{\perp}-H_{33} \mathbf{q}\left(\mathbf{k}_{\perp} \cdot \mathbf{q}\right)\right) p / \omega+v_{3} \mathbf{q}
$$

It is worth noting that even at normal incidence, with $\mathbf{k}_{\perp}=(0,0)$, the coupling still occurs from the term $v_{3} \mathbf{q}$. In order to illustrate this effect, Fig. 4 shows the absorption coefficient when the fluid is taken out of its principal directions. Also considering normal incidence and with $\mathcal{R}_{0} \equiv \mathcal{R}_{\Omega}$, a sole rotation around $\mathbf{e}_{3}$ cannot impact the acoustic properties of the fluid. First, the dependence on the rotation angle around $\mathbf{e}_{1}$ is shown in Fig.4(a), which is $\pi$-periodic. Then on Fig.4(b) the absorption coefficient varies as the cell is rotated around the $\mathbf{e}_{2}$ unit vector. 

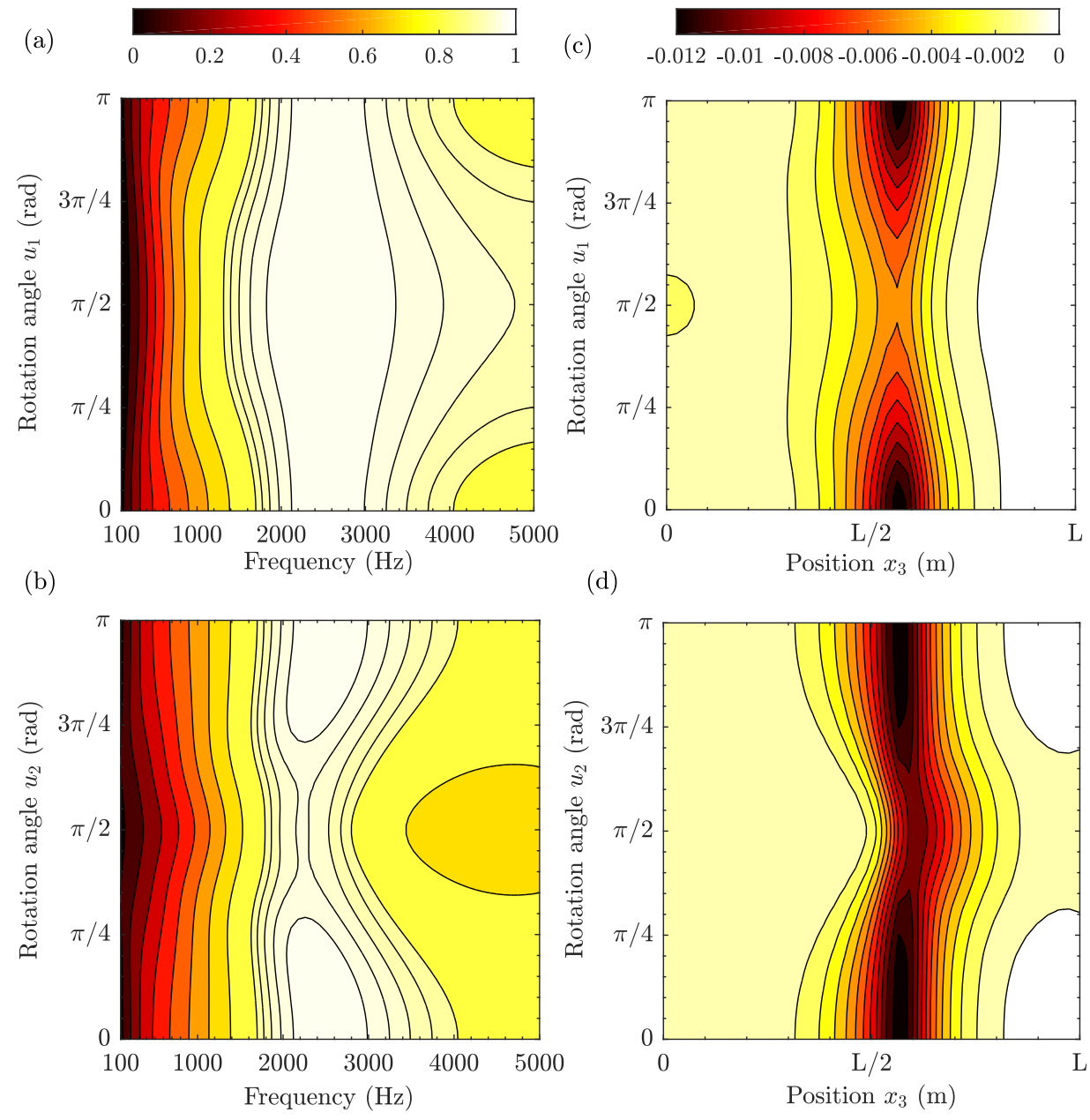

(d)

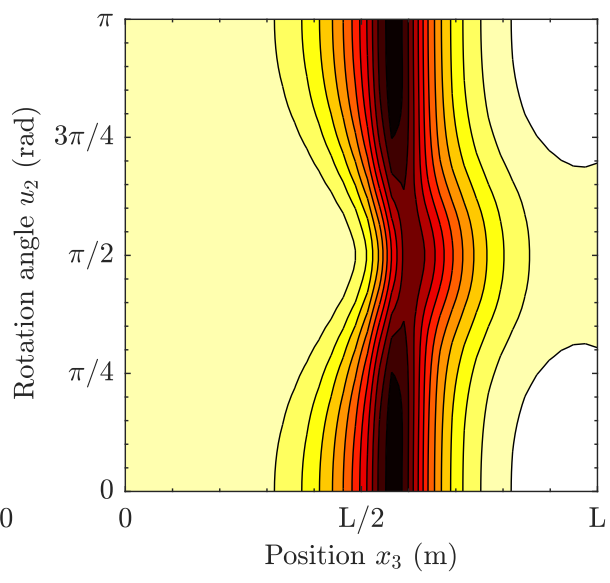

FIG. 4. [Color online] Absorption coefficient at normal incidence as a function of the circular frequency $\omega$, the rotation angle $u_{1}$ in (a) and $u_{2}$ in (b). Energy dissipation rate at normal incidence between $x_{3}=0$ and $L$ for rotation angles $u_{1}(\mathrm{c})$ and $u_{2}(\mathrm{~d})$, from 0 to $\pi$ at the perfect absorption frequency $f_{0}$.

As depicted in Eq. (35), the estimated dissipation rate directly depend on the rotations applied to the density tensor. Figures 4(c) and 4(d) display the estimated dissipation inside the domain $\Omega$, at frequency $f_{0}$. As previously, the dependence on the rotation angles $\left(u_{1}, u_{2}\right)$ affects the losses in the fluid, hence on the absorption properties. We notice that most of 
the energy losses in the domain are localized where the pore size becomes small, which is correlated to the total pressure profile in Fig.3(d).

\section{E. Diffuse field absorption}

Instead of a single wave with a specific incidence angle, one can also consider a diffuse field where all wave directions are present, but uncorrelated with the same intensity. The corresponding absorption coefficient accounts for the absorption averaged over all possible angles of incidence:

$$
\alpha_{d i f}(\omega)=\frac{1}{2 \pi} \int_{0}^{\pi} \int_{0}^{\pi} \alpha(\omega, \theta, \psi) \cos (\theta) \mathrm{d} \theta \mathrm{d} \psi
$$

with $(\theta, \psi) \in[0, \pi]^{2}$ and frequency $\omega$. The averaging process is done accounting for the solid angle associated to each direction of incidence, which induces the weight $\cos (\theta)$. This diffuse field absorption coefficient is shown in Fig. 5 as a function of frequency using 400 plane wave direction to compute the average. As pictured in Fig.5, the graded anisotropic materials is able to provide good diffuse absorption over a wide range of frequencies. However its absorption is limited at low frequencies. Unlike the absorption of the plane wave at normal incidence which is perfect around $2500 \mathrm{~Hz}$ (see Fig.3), the diffuse field case is unable to reach a perfect absorption. This is explained by the contributions of the plane waves with grazing incidence which can only be partially absorbed. But as oblique incidences weight a lot in this considerations, the anisotropic properties firmly impact the diffuse field absorption coefficient. 


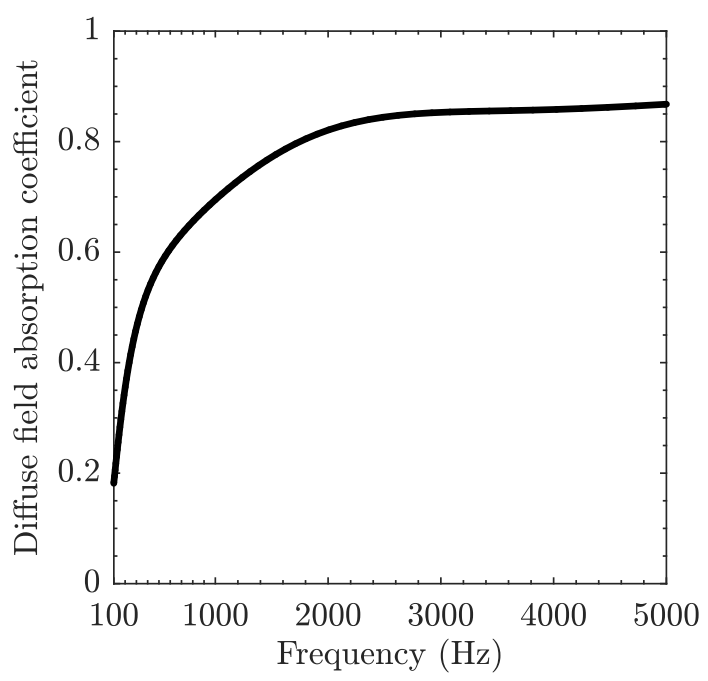

FIG. 5. Diffuse field absorption coefficient as a function of frequency.

\section{CONCLUSIONS}

In this work, the propagation of acoustic waves through a graded layer of anisotropic fluid has been modeled to calculate the transmission and reflection coefficients. This approach is applicable to a wide range of porous materials that are described by their effective bulk modulus and density tensor, and in this case is developed for non-symmetric heterogeneous systems. Three different numerical techniques have been presented and compared to solve for the sound field in such a layer. Two of the solution procedures account for the continuous macro-modulated effective properties of the anisotropic medium, and altogether show excellent agreement with the more traditional TMM approach. In addition, the knowledge of the pressure and velocity fields inside the anisotropic fluid provides useful insight into the losses occurring within the layer.

The dependence of the absorption coefficient with frequency (over the range $100 \mathrm{~Hz}-$ $5 \mathrm{kHz}$ ), angles of incidence and orientation of the micro-structure has been discussed in 
detail. All the results demonstrates the complex interplay between these parameters and the fact that the anisotropy plays a significant role in the absorption achieved by this kind of materials. The absorption of a diffuse field was also considered.

The use of anisotropic and heterogeneous materials drastically enhances the potential for efficient acoustic control in scattering and absorption problems. The next step on this topic would be to perform a full optimization of both the anisotropy and the heterogeneity of a porous layer, so as to maximize the acoustic absorption in specific applications.

\section{ACKNOWLEDGMENTS}

The authors gratefully acknowledge the support from ANR Chaire industrielle MACIA (ANR-16-CHIN-0002).

\section{REFERENCES}

${ }^{1}$ D. Lafarge, P. Lemarinier, J. F. Allard, and V. Tarnow, "Dynamic compressibility of air in porous structures at audible frequencies," The Journal of the Acoustical Society of America 102(4), 1995-2006 (1997) http://asa.scitation.org/doi/10.1121/1.419690 doi: $10.1121 / 1.419690$.

${ }^{2}$ J.-L. Auriault, C. Boutin, and C. Geindreau, Homogenization of coupled phenomena in heterogenous media (ISTE, London, 2009), oCLC: 699253480.

${ }^{3}$ C. Van der Kelen and P. Göransson, "Identification of the full anisotropic flow resistivity tensor for multiple glass wool and melamine foam samples," The Journal of the Acoustical 
Society of America 134(6), 4659-4669 (2013) http://asa.scitation.org/doi/10.1121/ 1.4824841 doi: $10.1121 / 1.4824841$.

${ }^{4} \mathrm{~V}$. Tarnow, "Measured anisotropic air flow resistivity and sound attenuation of glass wool," The Journal of the Acoustical Society of America 111(6), 2735-2739 (2002) http: //asa. scitation.org/doi/10.1121/1.1476686 doi: 10.1121/1.1476686.

${ }^{5} \mathrm{~V}$. Tarnow, "Compressibility of air in fibrous materials," The Journal of the Acoustical Society of America 99(5), 3010-3017 (1996) http://asa.scitation.org/doi/10.1121/ 1.414790 doi: $10.1121 / 1.414790$.

${ }^{6}$ B. Nennig, R. Binois, N. Dauchez, E. Perrey-Debain, and F. Foucart, "A transverse isotropic equivalent fluid model combining both limp and rigid frame behaviors for fibrous materials," The Journal of the Acoustical Society of America 143(4), 2089-2098 (2018) http://asa.scitation.org/doi/10.1121/1.5030925 doi: 10.1121/1.5030925.

${ }^{7}$ A. Terroir, L. Schwan, T. Cavalieri, V. Romero-García, G. Gabard, and J.-P. Groby, "General method to retrieve all effective acoustic properties of fully-anisotropic fluid materials in three dimensional space," Journal of Applied Physics 125(2), 025114 (2019) http://aip.scitation.org/doi/10.1063/1.5066608 doi: 10.1063/1.5066608.

${ }^{8}$ B. Brouard, D. Lafarge, and J.-F. Allard, "A general method of modelling sound propagation in layered media," Journal of Sound and Vibration 183(1), 129-142 (1995) https://linkinghub.elsevier.com/retrieve/pii/S0022460X8570243X doi: 10.1006/ jsvi.1995.0243. 
${ }^{9}$ A. Geslain, J. P. Groby, O. Dazel, S. Mahasaranon, K. V. Horoshenkov, and A. Khan, "An application of the Peano series expansion to predict sound propagation in materials with continuous pore stratification," The Journal of the Acoustical Society of America 132(1), 208-215 (2012) http://asa.scitation.org/doi/10.1121/1.4728188 doi: 10.1121/1. 4728188.

${ }^{10}$ G. Gautier, L. Kelders, J. P. Groby, O. Dazel, L. De Ryck, and P. Leclaire, "Propagation of acoustic waves in a one-dimensional macroscopically inhomogeneous poroelastic material," The Journal of the Acoustical Society of America 130(3), 1390-1398 (2011) http://asa. scitation.org/doi/10.1121/1.3605530 doi: 10.1121/1.3605530.

${ }^{11} \mathrm{C}$. Baron, "Matricant Peano series development to study elastic waves propagation in continuously varying properties materials," Ph.D. thesis, 2005.

${ }^{12}$ A. Shuvalov, O. Poncelet, and M. Deschamps, "General formalism for plane guided waves in transversely inhomogeneous anisotropic plates," Wave Motion 40(4), 413-426 (2004) https://linkinghub.elsevier.com/retrieve/pii/S0165212504000769 doi: 10.1016/ j.wavemoti.2004.02.008.

${ }^{13}$ L. De Ryck, W. Lauriks, Z. E. A. Fellah, A. Wirgin, J. P. Groby, P. Leclaire, and C. Depollier, "Acoustic wave propagation and internal fields in rigid frame macroscopically inhomogeneous porous media," Journal of Applied Physics 102(2), 024910 (2007) http://aip.scitation.org/doi/10.1063/1.2752135 doi: 10.1063/1.2752135.

${ }^{14}$ L. De Ryck, J.-P. Groby, P. Leclaire, W. Lauriks, A. Wirgin, Z. E. A. Fellah, and C. Depollier, "Acoustic wave propagation in a macroscopically inhomogeneous porous 
medium saturated by a fluid," Applied Physics Letters 90(18), 181901 (2007) http: //aip.scitation.org/doi/10.1063/1.2431570 doi: 10.1063/1.2431570.

${ }^{15}$ J. Lundstedt and M. Norgren, "Comparison between Frequency Domain and Time Domain Methods for Parameter Reconstruction on Nonuniform Dispersive Transmission Lines," Progress In Electromagnetics Research 43, 1-37 (2003) http://www.jpier.org/PIER/ pier .php?paper=0302031 doi: 10.2528/PIER03020301.

${ }^{16} \mathrm{M}$. Norgren, "General scheme for electromagnetic reflection and transmission for composite structures of complex materials," IEE Proceedings - Microwaves, Antennas and Propagation 142(1), 52 (1995) https://digital-library.theiet.org/content/journals/10. 1049/ip-map_19951533 doi: 10.1049/ip-map: 19951533.

${ }^{17}$ O. Forslund and S. He, "Electromagnetic Scattering from an Inhomogeneous Grating Using a Wave-Splitting Approach," Progress In Electromagnetics Research 19, 147-171 (1998) http://www . jpier . org/PIER/pier . php?paper=971015 doi: 10.2528/PIER97101500.

${ }^{18}$ G. N. Borzdov, "Frequency domain wave-splitting techniques for plane stratified bianisotropic media," Journal of Mathematical Physics 38(12), 6328-6366 (1997) http: //aip.scitation.org/doi/10.1063/1.532216 doi: 10.1063/1.532216.

${ }^{19} \mathrm{R}$. Krueger and R. Ochs, "A green's function approach to the determination of internal fields," Wave Motion 11(6), 525-543 (1989) https://linkinghub.elsevier.com/ retrieve/pii/0165212589900243 doi: 10.1016/0165-2125(89)90024-3.

${ }^{20}$ M. C. Pease, Methods of Matrix Algebra. (Elsevier NetLibrary, Incorporated [distributor, New York; Boulder, 1965), http://www.sciencedirect.com/science/bookseries/ 
374

375

376

377

378

00765392/16, oCLC: 944502142.

${ }^{21}$ R. A. Horn and C. R. Johnson, Matrix analysis, second edition, corrected reprint ed. (Cambridge University Press, New York, NY, 2017).

${ }^{22}$ M. Bruneau, Fundamentals of acoustics (ISTE Ltd, London ; Newport Beach, CA, 2006), oCLC: ocm68711955. 